\title{
Estimation of Effective Thermal Conductivity of Two-Phase Material for Hollow Circular Model
}

\author{
Prateek Kumar Sahu ${ }^{1}$, Nisha Netam $^{2 *}$, Lal Chandra Shah ${ }^{3}$ \\ ${ }_{1,2}$ Department of Mechanical Engineering, National Institute of Technology Raipur, Chhattisgarh, 492010, India \\ ${ }^{3}$ Department of Mechanical Engineering, Shri Shankaracharya Institute of Technology and Management, Bhilai, 490020, India
}

\begin{abstract}
Two-phase materials are commonly used in engineering application because of its various properties like strength, thermal conductivity, durability and toughness etc. Effective thermal conductivity (ETC) of two-phase material is the fundamental property to predict its thermal performance. Various geometry (spheres, cylinders, irregular particles) have been considered by researchers for calculating ETC of two-phase materials. Due to complex structure, hollow circular cylinder geometry is not reported yet. In this paper, two-dimensional periodic two-phase system, with hollow circular cylinder shape is considered for calculating ETC. In present work unit cell approach method is used to derive collocated parameters model for estimation of ETC. Hollow circular cylinder model with $\Psi=0.2$ gives good result for estimating ETC with average percentage error of $6.46 \%$.
\end{abstract}

Keywords: Effective Thermal Conductivity, Two-Phase Material, Unit cell approach, Hollow circular model.

\section{Introduction}

A two-phase material consists of two phases with distinct chemical and physical properties. Two phases can be solid-solid, solid-fluid and fluid-fluid. Phases are named as continuous and dispersed, and categorized into various systems based on its characteristics. Two-phase materials are commonly used in application of engineering field because of its various properties like strength, thermal conductivity, durability and toughness etc. Effective thermal conductivity (ETC) of two-phase material (TPM) is the fundamental property to predict its thermal performance. TPM are categorized into the homogeneous or heterogeneous structure. In homogeneous systems, chemical composition will be same throughout the matrix whereas, in the heterogeneous system, chemical composition will be different throughout the matrix.

Two reference model proposed by [1], for calculating ETC are series model and parallel model. Where in series model phases are in series with the heat direction and in parallel model phases are in parallel with heat direction.

$$
\begin{aligned}
& \mathrm{k}_{\mathrm{e}}=\frac{\mathrm{k}_{\mathrm{s}} \mathrm{k}_{\mathrm{f}}}{\varepsilon \cdot \mathrm{k}_{\mathrm{s}}+(1-\varepsilon) \cdot \mathrm{k}_{\mathrm{f}}} \quad \text { Minimum (series) } \\
& \mathrm{k}_{\mathrm{e}}=\varepsilon \cdot \mathrm{k}_{\mathrm{f}}+(1-\varepsilon) \cdot \mathrm{k}_{\mathrm{s}} \quad \text { Maximum (parallel) }
\end{aligned}
$$

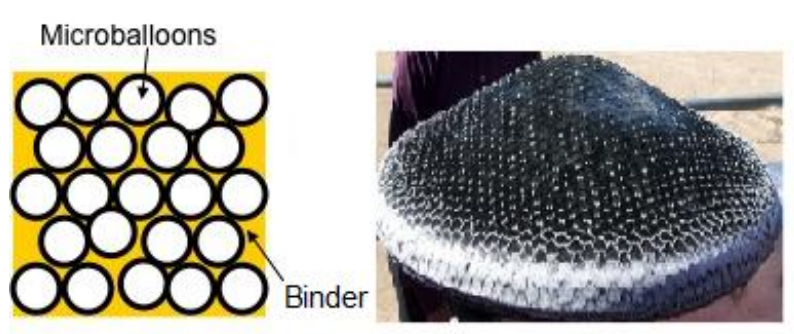

Fig 1 Schematic of two-phase material [7].

A model for calculating ETC TPM has been proposed by [2]. In this model suspension of solid sphere are considered which are not in contact.

$$
\mathrm{K}=\frac{1+2 \beta v}{1-\beta v}
$$

where $\beta$ is given as:

$$
\beta=\frac{\alpha-1}{\alpha+2}
$$

[3] Developed the model, with the effect of (i) conduction through solid phase, contact surfaces and in voids through the fluid medium (ii) heat transfer through radiation, for estimating ETC of two-phase material. A mathematical expression for the ETC of a packed bed with the inclusion of the effect of thermal radiation, dependency on pressure, particle flattening, shaping and size distribution, along with particle contact for a cylindrical unit cell that contains a spherical particle is presented [4]. [5] Reported an analytical expression based on unit cell approach for calculating ETC of two- 
phase material for the square cylinder, circular cylinder, solid cube and hollow cube. [6] Presented an analytical expression based on unit cell approach, for hexagonal and octagonal cinder model for estimating ETC. Results show average deviation of $\pm 15.58 \%$ with experiments results.

Various geometry (spheres, cylinders, irregular particles) have been considered by researchers for calculating ETC of two-phase materials. Due to the complex structure, hollow circular cylinder geometry is not reported yet. In this paper, two-dimensional periodic two-phase system, with hollow circular cylinder shape has been considered for deriving an algebraic equation for calculating ETC. A unit cell approach is used to calculate the ETC.

\section{Methodology}

Parameters on which ETC of two-phase materials depends are conductivity ratio $(\alpha)$ and concentration $(v)$, contact resistance, convective heat transfer, radiative heat transfer and Knudsen effect. It also depends on particle size, shape, location, size distribution and orientation. Unit cell approach based on collocated parameter model is used to derive the expression to calculate the ETC for hollow circular cylinder model.

The ETC of the two-dimensional, hollow circular cylinder is determined for Fig. 2, with the inner radius of " $b$ ", outer radius of " a " and connecting bar width of " c ". Due to structure symmetry, quartern of the structure is taken as an unit cell and is shown in Fig. 3.

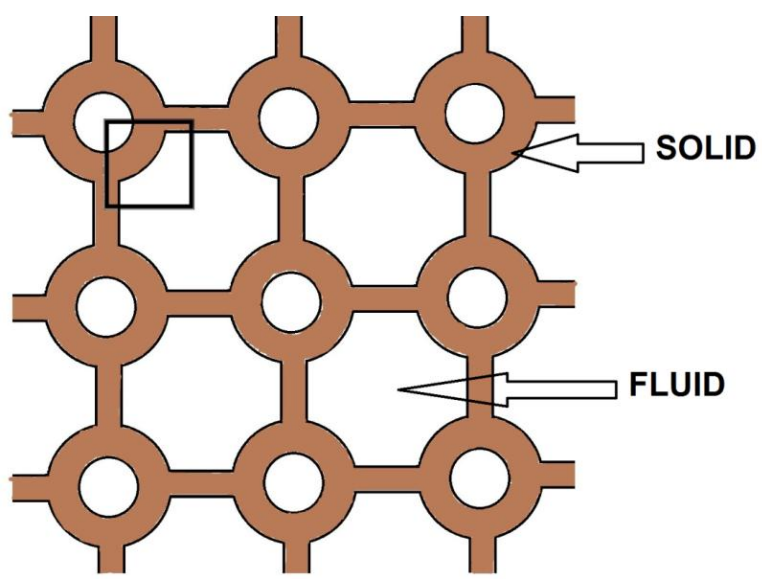

Fig. 2 Collocated Hollow Cicular Cylinder Model

The unit cell is separated into four rectangular layers as shown in Fig. 3, perpendicular to the direction of heat. Based on series approach TC of each layer is acquired. Every layer has both fluid and also solid phase in the unit cell.

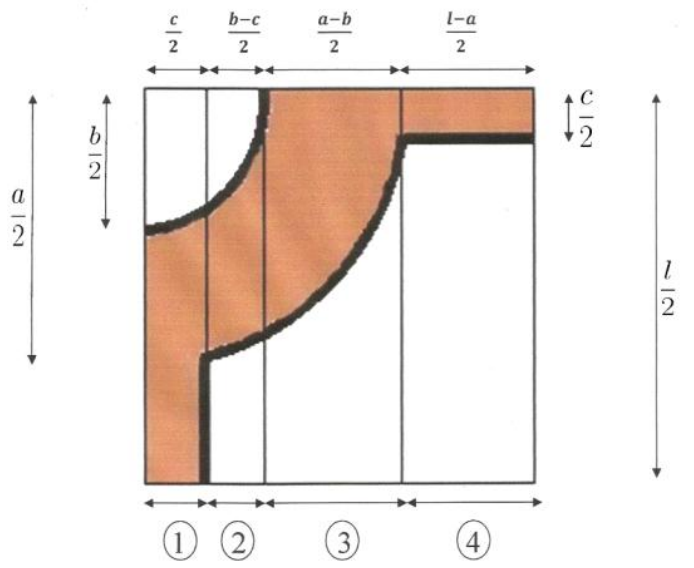

Fig. 3 Unit Cell of Hollow Circular Cylinder Model

Total resistance in unit cell is:

$$
\mathrm{R}_{\text {total }}=\mathrm{R}_{1}+\mathrm{R}_{2}+\mathrm{R}_{3}+\mathrm{R}_{4}
$$

Here $\mathrm{R}_{1}, \mathrm{R}_{2}, \mathrm{R}_{3}$ and $\mathrm{R}_{4}$ are the thermal resistance of layer $1,2,3$ and 4 respectively.

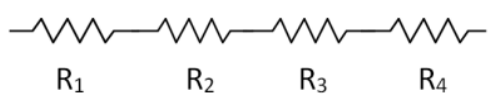

Fig.4Thermal Resistance

Now thermal resistance for layer 1 is calculated as:

$$
\begin{gathered}
\text { Thermal Resistance }=\frac{\text { Thickness of rectangual strip }}{\text { Thermal conductivity X Cross secion area of strip }} \\
\qquad \begin{array}{c}
\frac{1}{R_{1}}=\left(\frac{K_{s f 1} X \text { Area }}{\text { Thickness }}\right)+\left(\frac{K_{s} X \text { Area }}{\text { Thickness }}\right) \\
\frac{1}{R_{1}}=\left(\frac{K_{s f 1} l(b / 2)}{c / 2}\right)+\left(\frac{K_{s} l(l-b) / 2}{c / 2}\right) \\
R_{1}=\frac{c}{K_{s f 1} l b+K_{s} l(l-b)}
\end{array}
\end{gathered}
$$

Similarly, thermal resistance for layer 1, 2, 3 and 4 are:

$$
\begin{gathered}
R_{2}+R_{3}=\left[\left\{\frac{b-c}{K_{s f 2} l c}+\frac{a-b}{K_{s} l c}\right\}^{-1}+\frac{2 K_{s} l}{\pi}+\frac{2 K_{s f 2} l}{\pi-2}+\frac{K_{s f 2} l(l-a)}{a-c}\right]^{-1} \\
R_{4}=\frac{l-a}{K_{s} c l+K_{s f 4} l(l-c)}
\end{gathered}
$$

Total resistance in unit cell is:

$$
\begin{aligned}
R_{\text {total }}= & \frac{c}{K_{s f 1} l b+K_{s} l(l-b)}+\left[\left\{\frac{b-c}{K_{s f 2} l c}+\frac{a-b}{K_{s} l c}\right\}^{-1}+\frac{2 K_{s} l}{\pi}+\frac{2 K_{s f 2} l}{\pi-2}+\frac{K_{s f 2} l(l-a)}{a-c}\right]^{-1}+ \\
& \frac{l-a}{K_{s} c l+K_{s f 4} l(l-c)}
\end{aligned}
$$

The value of thermal conductivity at solid fluid interface for layer 1, 2, 3 and 4 is determined based on a series layer model: 


$$
\begin{gathered}
\phi_{1}=\frac{K_{s f 1}}{K_{s}}=\frac{\alpha}{1+(\alpha-1) \delta \varepsilon} \\
\phi_{2}=\frac{K_{s f 2}}{K_{s}}=\frac{2 \alpha}{(\alpha-1) \delta \varepsilon+(1-\varepsilon)(1-2 \alpha)} \\
\phi_{3}=\frac{K_{s f 3}}{K_{s}}=\frac{2 \alpha}{(\psi+1) \varepsilon+\alpha(2-\varepsilon-\delta \varepsilon)} \\
\phi_{4}=\frac{K_{s f 4}}{K_{s}}=\frac{\alpha}{(1-\varepsilon \psi) \alpha+\varepsilon \alpha}
\end{gathered}
$$

Where,

Conductivity ratio $(\alpha)=\frac{K_{s}}{K_{f}}$

Length Ratio $(\varepsilon)=\frac{\alpha}{l}$

Contact Ratio $(\psi)=\frac{c}{a}$

Hollow Shape Factor $(\delta)=\frac{b}{a}$

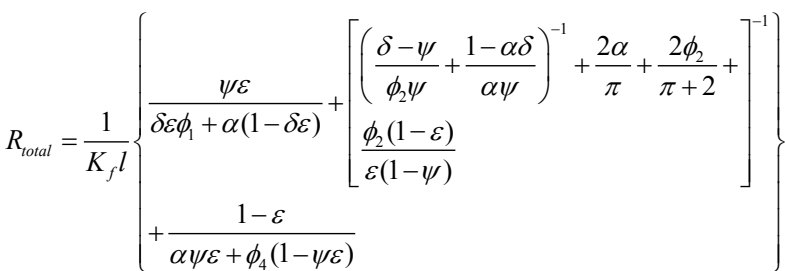

$$
\begin{gathered}
K_{\text {eff }}=\frac{1}{R_{\text {total }} l} \\
K=\frac{K_{\text {eff }}}{K_{f}}=\left\{\begin{array}{l}
\left.\frac{\psi \varepsilon}{\delta \varepsilon \phi_{1}+\alpha(1-\delta \varepsilon)}+\left[\begin{array}{l}
\left.\left(\frac{\delta-\psi}{\phi_{2} \psi}+\frac{1-\alpha \delta}{\alpha \psi}\right)^{-1}+\frac{2 \alpha}{\pi}+\frac{2 \phi_{2}}{\pi+2}+\right]_{2}^{-1} \\
\frac{\phi_{2}(1-\varepsilon)}{\varepsilon(1-\psi)}
\end{array}\right]\right\} \\
+\frac{1-\varepsilon}{\alpha \psi \varepsilon+\phi_{4}(1-\psi \varepsilon)}
\end{array}\right]^{-1}
\end{gathered}
$$

$K=f(\alpha, \delta, \psi, \varepsilon)$

Here $\mathrm{K}$ is the non-dimensional thermal conductivity (TC) for collocated parameter of hollow circular cylinder it is a function of conductivity ratio, hollow shape factor, length ratio and contact ratio.

The solid phase portion of the unit cell is indicated in terms of concentration ( $v$ ), and is given by:

$v=\frac{\text { Area of the solid phase }}{\text { Total area of the unit cell }}$

$$
v=\frac{\pi}{4} \varepsilon^{2}\left(1-\delta^{2}\right)+2 \varepsilon \psi(1-\varepsilon)
$$

\section{Results and discussion}

Non-dimensional TC is calculated for glass wool $(\alpha=10)$ using Eq. (16), and results are plotted between nondimensional TC and concentration (v) for different contact ratio (c/a). Fig 5 shows the variation of nondimensional TC with concentration $(v)$ for hollow circular model with hollow shape factor $(\delta=0.25)$ and different contact ratio $(\mathrm{c} / \mathrm{a}=0,0.1,0.2)$. Fig. 6 shows the variation of non-dimensional TC with concentration $(v)$ for hollow circular cylinder model with hollow shape factor of $\boldsymbol{\delta}=0.75$ and different contact ratio $(\mathrm{c} / \mathrm{a}=0,0.1$, 0.2 ). Variation of temperature on non-dimensional TC is presented in Fig. 7, with temperature non-dimensional $\mathrm{TC}$ is increases.

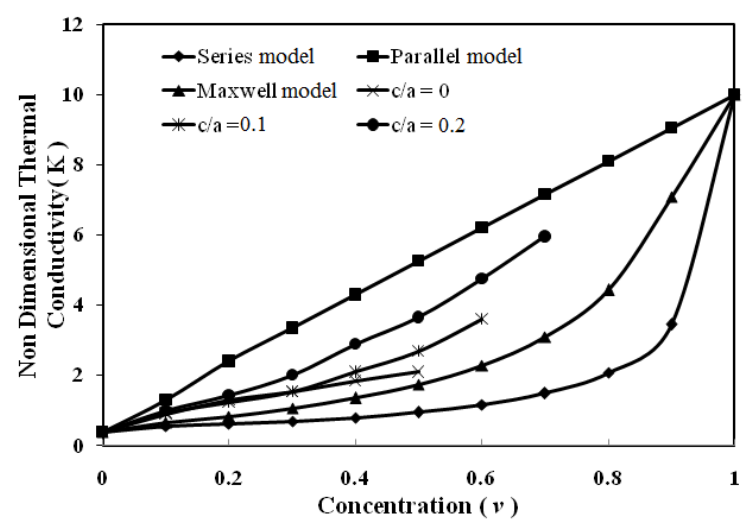

Fig. 5 Variation of non-dimensional TC with concentration ( v ) for hollow circular cylinder $(\alpha=10 \& \delta=0.25)$.

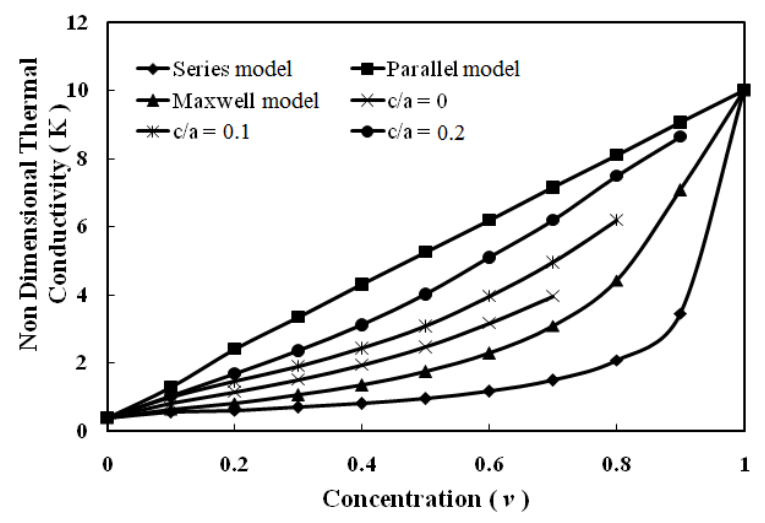

Fig. 6 Variation of non-dimensional TC with concentration (v) for hollow circular cylinder $(\alpha=10 \& \delta=0.75)$.

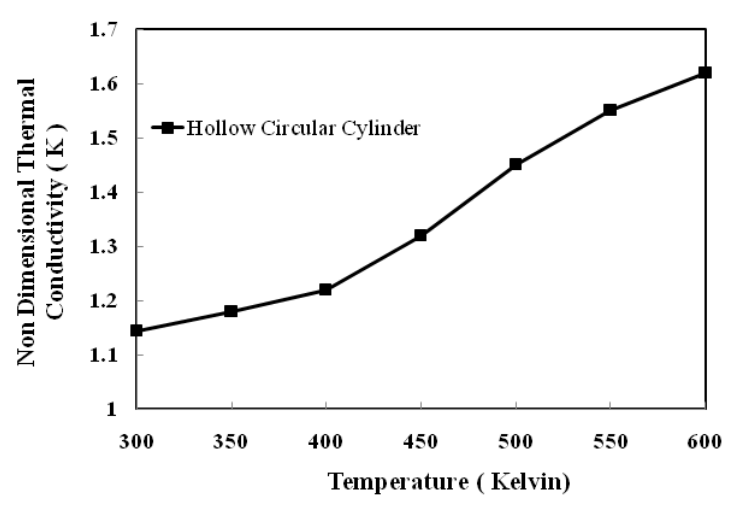

Fig.7 Effect of temperature on the non-dimensional TC of two-dimensional hollow circular cylinder $(\delta=0.5, \Psi=0.2$ and $\varepsilon=0.3$ ). 
Table 1 Result comparison with experimental value

\begin{tabular}{lccll}
$\begin{array}{l}\text { Two-Phase } \\
\text { Material }\end{array}$ & $\boldsymbol{\alpha}$ & $\mathbf{K}_{\text {exp }}$ & $\mathbf{K}_{\text {eff }}$ & $\begin{array}{c}\% \\
\text { Deviation }\end{array}$ \\
\hline Glass Wool & 10 & 1.2 & 1.1437 & 4.69 \\
$\begin{array}{l}\text { Alumina Air } \\
\text { Air and }\end{array}$ & 1.6 & 1.7008 & 6.3 \\
Sand & 40 & 2.1 & 2.2768 & 8.4 \\
\hline & & Average & 6.46 \\
\hline
\end{tabular}

\section{Conclusion}

The non-dimensional TC of the two-phase material $(\alpha=10)$ has been calculated for concentration range $(0.2$ $\leq \mathrm{v} \leq 0.9$ ). Results of the present model lie, between the standard model (parallel and series) and above the Maxwell model. Hollow circular cylinder model with $\Psi$ $=0.2$ gives the good result for estimating thermal conductivity with average percentage error $6.46 \%$ as shown in Table 1. The present model can be used to calculate the ETC of two-phase material for concentrations $(0 \leq \mathrm{v} \leq 0.9)$ for hollow circular geometry.

\section{Nomenclature}

$\mathrm{K}_{\mathrm{sf}}$ Thermal conductivity at solid fluid interface

$\mathrm{K}_{\mathrm{s}} \quad$ Thermal conductivity of solid

$\mathrm{K}_{\mathrm{f}} \quad$ Thermal conductivity of fluid

$\phi \quad$ Solid fraction

v Concentration

\section{References}

1. I. H. Tavman, Int. Comm. Heat Mass Transfer, 23, No. 2, pp. 169-176 (1996).

2. J. C. Maxwell, A Treatise on Electricity and Magnetism, Clarendon Press, Oxford, p. 365 (1873).

3. S. Imura, E. Takegoshi Heat Transfer-Japan, 1326(1974).

4. P. Zehner, E. U. Schlunder Chemical Engineering Technology, 42, (1970).

5. K. S. Reddy, P. Karthikeyan, Heat Transfer Engineering, 30, Iss. 12, (2009).

6. A. P Senthil Kumar, V. Prabhu Raja, P. Karthikeyan, Journal of scientific and industrial research, 69, pp 356-364 (2010).

7. G. Gladysz, K. K. Chawla, VOIDS IN MATERIALS From Unavoidable Defects to Designed Cellular Materials, Elsevier (2015). 